\title{
Quality of hydro-alcoholic products used in Senegal: pilot study
}

\author{
Souleymane AIDARA ${ }^{1,2^{*}}$, Assane Dieng ${ }^{3,}$ Amadou DIOP ${ }^{2}$, Maguette DIEYE ${ }^{3}$, \\ Pape Matar MBAYE ${ }^{1}$, Mamadou Moustapha SEYE ${ }^{1}$, Mor FALL ${ }^{1}$, Adama DIEDHIOU ${ }^{3,4}$ \\ and Djibril FALL ${ }^{3,4}$
}

\author{
${ }^{I}$ Pharmacy Hospital Dantec, Dakar, Senegal. \\ ${ }^{2}$ Laboratories of Analytic Chemistry and Bromatology FMPO, UCAD, Dakar Senegal. \\ ${ }^{3}$ National Laboratory for Drug Quality Control, Dakar, Senegal. \\ ${ }^{4}$ Laboratory of Physical Chemistry, Therapeutic Chemistry, FMPO UCAD, Dakar, Senegal. \\ *Auteur correspondant ; E-mail : souleyaidara66@gmail.com ; Tel :775085182.
}

Received: 09-01-2021

Accepted: $22-06-2021$

Published: 30-06-2021

\begin{abstract}
Antisepsis and disinfection have always played an important role in the fight against infectious diseases. The use of these products has been effective in breaking the chain of transmission of microorganisms. Today, with the advent of COVID-19, the main recommendations are, among other things, physical distance and the use of antiseptic products, including hydro-alcoholic products. In Senegal, with poor regulations on the acquisition and distribution of antiseptics and disinfectants, this situation has led to a proliferation of antiseptics and disinfectants on the national market. this work aimed to study the quality of hydroalcoholic products found in the trade. We evaluated ten samples of hydro-alcohol products collected during the month of March 2020. Physical and microbiological controls were performed at the National Drug Control Laboratory. The alcohol content of the samples ranged from 63 to $85 \%$ and were consistent with WHO recommendations except for samples E3 and E5. The pH values varied between 4.02 and 6.64 and the densities of the hydro-alcoholic gel samples ranged from 0.84 to $0.92 \mathrm{~g} / \mathrm{cm}^{3}$; E2, E4, E5, E6 samples had densities greater than $0.89 \mathrm{~g} / \mathrm{cm}^{3}$. The samples of hydro-alcoholic products all conformed to the sterility test and no microbiological contamination was observed. Antimicrobial activities of the hydro-alcoholic samples tested ranged from 58.3 to $100 \%$ with two samples showing no activity (E2 and E5). In view of the results of this study, it would be relevant to expand and deepen investigations by a significant increase in the number of samples and by carrying out as complete an analysis as possible.
\end{abstract}

(C) 2021 International Formulae Group. All rights reserved.

Keywords: Hydro-alcoholic products; quality control; hand hygiene.

\section{INTRODUCTION}

Antisepsis and disinfection have always played an important role in the fight against infectious diseases. The use of antiseptic and disinfectant products has been effective in breaking the chain of transmission of microorganisms such as viruses, bacteria and fungi (Chabot Daval, 2010). During the 2009 influenza A (H1N1) pandemic, public health measures had to be put in place to prevent and limit the transmission of the virus in the population (Afssaps, 2010). One of the main measures to fight this scourge was the use of antiseptics and disinfectants (Travkine, 2012). 
Today, with the COVID-19 pandemic, the main recommendations include physical distance and the use of hydro-alcoholic products (Goetz, 2004; Meunier et al., 2012).

As a result of this pandemic, various forms of hydro-alcoholic products from a multitude of manufacturers have been put on the market as a consequence of the lack of a deficient regulation concerning the acquisition and distribution of antiseptics and disinfectants. In Senegal, no studies have been conducted to evaluate the efficacy of hydroalcoholic products. The aim of this work was to study the quality of hydroalcoholic products on the physico-chemical and antimicrobial activities level found in the trade.

\section{MATERIALS AND METHODS}

\section{Framework for the study}

Samples of hydro-alcoholic products were collected in different health structures (Table 1). The physicochemical and microbiological controls were carried out at the National Drug Control Laboratory.

\section{Materials}

- The material used for physical and chemical analyses consists of conventional laboratory glassware and consumables. It also includes equipment such as: magnetic agitator, alcoholmeter, $\mathrm{pH}$-meter (Metrohm, Switzerland) and a densitometer (05 DENSI 75 Germany).

- For microbiological analyses, the equipment included:

- Culture media: Mueller Hinton (MH), Thiogluconate Broth (BT); Consumables (petri dishes, discs, swabs, test tubes, vials, pipettes, tweezers, wash bottle, rack, test tubes and an oven).

- Reagents: sterile distilled water, sterile physiological water;

- Reference strain: Staphylococcus aureus ATCC 29293.

\section{Physico-chemical control}

Physical-chemical control consisted of determining the alcoholic degree (proportion of ethanol, contained in a hydroalcoholic product at a temperature of $20{ }^{\circ} \mathrm{C}$ ) using an alcoholmeter and making dilutions using the Gay Lussac table for hydro-alcoholic gels, determining $\mathrm{pH}$ and density.

\section{Sterility test of hydroalcoholic products}

For this test, we put $1 \mathrm{ml}$ of hydroalcoholic product sample in a liquid culture medium (Thiogluconate broth) that was incubated at $37{ }^{\circ} \mathrm{C}$ for 18 to 24 hours. The presence of turbidity visible to the naked eye in a sample of hydroalcoholic product incubated after 18 to 24 hours in thiogluconate broth is considered non-sterile.

\section{Antimicrobial activity test of hydro- alcoholic products}

This method consists in determining the antimicrobial activity of hydro-alcoholic products by comparing the number of colonyforming units (CFU) appearing on inoculated media in the presence of disinfectant and control media containing no disinfectant.

\section{Preparation of the inoculum 0.5 Mac Farland}

Starting from a young (18-24h) culture of the reference strain (Staphylococcus aureus ATCC 29293) 2 to 3 colonies are taken which are introduced into a test tube containing sterile physiological water. The density of the solution is measured with a Mac Farland densitometer and adjusted to a value of 0.5 on the Mac Farland scale.

\section{Contamination of sterile gloves}

A sterile glove is contaminated with 1 $\mathrm{ml}$ of the initially prepared 0.5 Mac Farland inoculum. The glove surface is rubbed and waited 30 seconds to ensure that the contamination is effective. We add $3 \mathrm{ml}$ of hydro-alcoholic products and then rub our hands for 30 seconds to allow good productgerm contact.

\section{Seeding of Thiogluconate Broth}

Using a swab, a sample is taken from the contaminated gloves with added disinfectant and introduced into the Thiogluconate broth medium and incubated at $37^{\circ} \mathrm{C}$ in the oven for 18 to 24 hours. Then two further samples were taken from the contaminated gloves without the addition of 
disinfectant and the uncontaminated gloves which are used as positive and negative controls.

A total of five tubes of Thiogluconate broth were prepared for each sample of hydroalcoholic products and distributed as follows:

- Tube 1 : broth + sterile glove (negative control) ;

- Tube 2: broth + contaminated sterile glove (positive control);

- Tube 3: BT broth alone (sterility control medium);

- Tube 4: broth $+3 \mathrm{ml}$ of hydro-alcoholic product (gel sterility control);

- Tube 5: broth + contaminated sterile glove $+3 \mathrm{ml}$ of hydro-alcoholic product (activity test).

Seeding of MH agar (Mueller Hinton)

A dilution series is carried out with the BT medium after 18 to 24 hours incubation by taking $10 \mu \mathrm{l}$ of BT which are introduced in $1000 \mu \mathrm{l}$ of physiological water. A series of three dilutions is carried out from the solution obtained by taking $10 \mu \mathrm{l}$ of the most concentrated diluted solution each time and introducing $1000 \mu \mathrm{l}$ of physiological water until the working solution used to inoculate the MH agar for bacterial enumeration is obtained. This inoculation is done using a calibrated 10 $\mu 1$ handle. Incubation was done at $37^{\circ} \mathrm{C}$ in an oven for 24 hours. The enumeration is performed by counting the colonies on the petri dish. The percentage antibacterial activity is given by the following formula:

$\mathrm{N}(\mathrm{CFU} / \mathrm{ml})=$ number of colonies counted .

\section{$A=(N$ positive control $-\mathbf{N}$ sample $) / N$ positive control $X 100$}

$\mathrm{A}=$ activity $(\%)$

$\mathrm{N}$ positive control $=$ number of positive control colonies

$\mathrm{N}$ sample $=$ number of colonies in the sample.

Table 1: Lots of hydro-alcoholic products collected.

\begin{tabular}{lcc}
\hline Sample & \multicolumn{1}{c}{ Name } & Manufacturer \\
\hline E1 & Hydroalcoholic solution & CHN Aristide Le Dantec Hospital Pharmacy \\
\hline E2 & Hydro-alcoholic gel & A pharmacy in the department of Dakar \\
\hline E3 & Hydro-alcoholic gel & A pharmacy in the department of Dakar \\
\hline E4 & Hydro-alcoholic gel & A pharmacy in the department of Dakar \\
\hline E5 & Hydro-alcoholic gel & A pharmacy in the department of Dakar \\
\hline E6 & Hydro-alcoholic gel & A pharmacy in the department of Dakar \\
\hline E7 & Hydro-alcoholic gel & CHN Aristide Le Dantec Hospital Pharmacy \\
\hline E8 & Hydro-alcoholic gel & CHN Aristide Le Dantec Hospital Pharmacy \\
\hline E9 & Hydro-alcoholic gel & CHN de Fann Hospital Pharmacy \\
\hline E10 & Hydro-alcoholic gel & A pharmacy in the department of Saint Louis \\
\hline
\end{tabular}




\section{RESULTS}

The alcohol contents of the samples of hydroalcoholic products varied between 63 and $85 \%$ (Table 2).

The $\mathrm{pH}$ of the samples ranged from 4.02 to 6.64 (Table 2), with only the E2 sample having an acidic $\mathrm{pH}$ value.

The densities of the hydroalcoholic gel samples ranged from 0.84 to $0.92 \mathrm{~g} / \mathrm{cm}^{3}$; samples E2, E4, E5, E6 had densities higher than $0.89 \mathrm{~g} / \mathrm{cm}^{3}$ (Table 2).
Concerning the sterility of the hydroalcoholic products, all the samples were in conformity with and no contamination of bacterial origin was observed (Figure 1).

The antimicrobial activities of the hydro-alcoholic product samples tested ranged from 58.3 to $100 \%$ with two samples showing no activity (E2 and E5). The hydro-alcoholic solution showed $100 \%$ higher activity than the hydro-alcoholic gels (Figure 2).

Table 2: Results of the physico-chemical parameters (Alcoholic degree $\mathrm{pH}$ and density) of the samples of hydroalcoholic products.

\begin{tabular}{|c|c|c|c|c|}
\hline Samples & $\begin{array}{c}\text { Alcoholic } \\
\text { degree }(\%)\end{array}$ & pH & $\begin{array}{r}\text { Density } \\
\left(\mathrm{g} / \mathrm{cm}^{3}\right) \\
\end{array}$ & Standard \\
\hline E1 & 83 & 6,64 & - & \multirow{3}{*}{$\begin{array}{l}\text { Alcoholic degree (\%) } \\
75-80 \% \text { (WHO Standard) }\end{array}$} \\
\hline E2 & 63 & 4,02 & $0,91 *$ & \\
\hline $\mathbf{E 3}$ & $84^{*}$ & 6,45 & 0,85 & \\
\hline E4 & 63 & 6,4 & $0,91^{*}$ & $\mathbf{p H} \quad(6,0-7,0)$ \\
\hline E5 & $85^{*}$ & 6,2 & $0,92 *$ & \\
\hline E6 & 68 & 6,27 & $0,90 *$ & Density $\left(\mathrm{g} / \mathrm{cm}^{3}\right)$ \\
\hline E7 & 75 & 6,5 & 0,84 & $(0,85-0,89)$ \\
\hline E8 & 72 & 6,7 & 0,89 & \\
\hline E9 & 73 & 6,45 & 0,88 & \\
\hline E10 & 67 & 6,02 & 0,89 & \\
\hline
\end{tabular}

*not in conformity
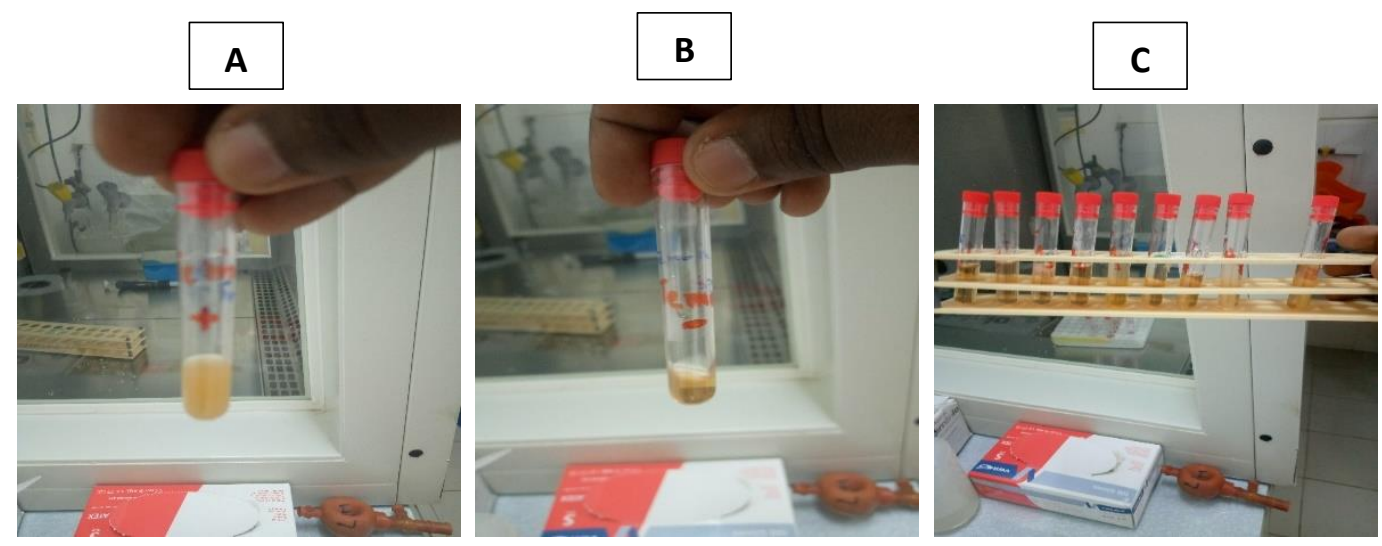

Figure 1: Sterility test results for hydro-alcoholic products.

(A: positive control; B: negative control; C: samples). 


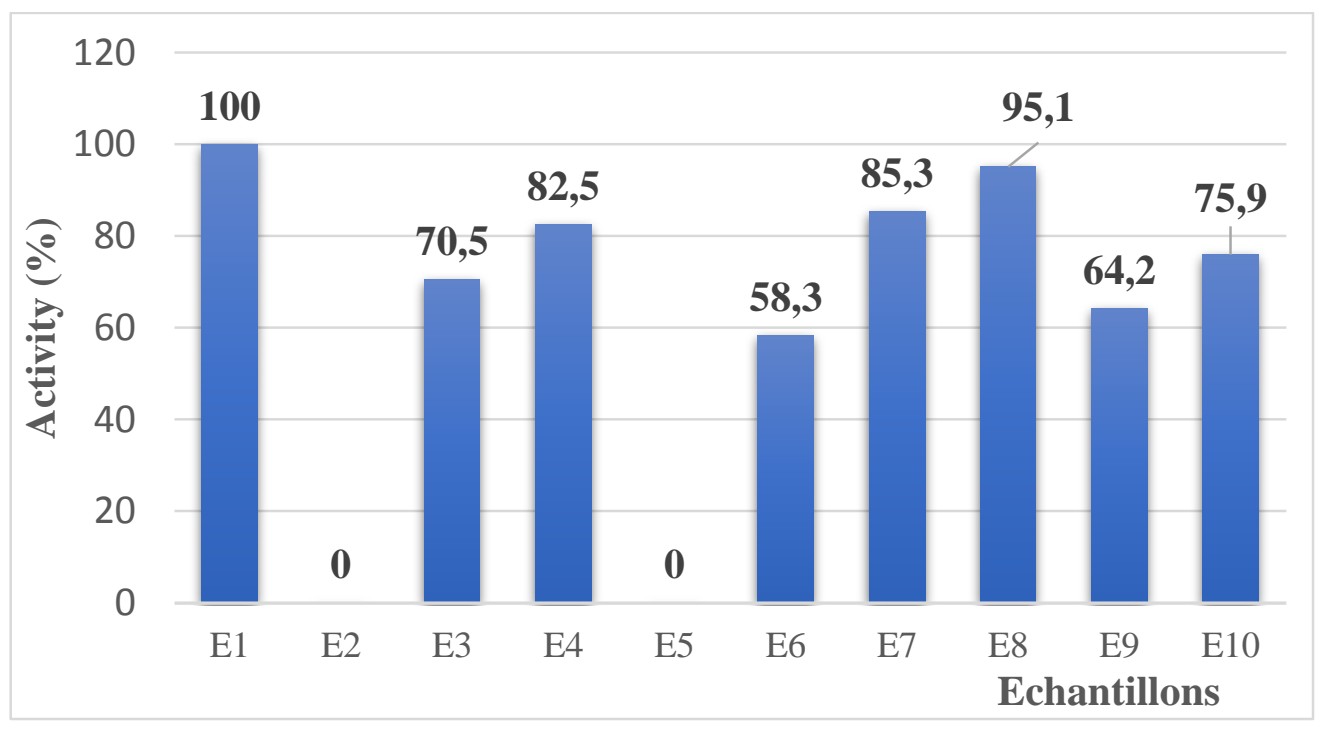

Figure 2: Results of the antimicrobial activity test of hydroalcoholic products.

\section{DISCUSSION}

The alcoholic degree is an important parameter in the evaluation of the quality of hydroalcoholic products. Indeed, ethanol is capable of inhibiting the growth of microorganisms even at low concentrations by acting on the wall, membrane and envelope of microorganisms (Rouzic et al., 2011). The activity of alcohol is concentration-dependent and it has been shown that hydroalcoholic products containing $60-80 \%$ ethanol is the most effective (WHO, 2005; Lejeune, 2008). In addition, both under- and overdosing can affect the antimicrobial activity of hydroalcoholic products. Indeed, it has been proven that anhydrous alcohol (100\%) has no antimicrobial activity (Samake, 2012). The majority of samples showed an antimicrobial activity higher than the $50 \%$ recommended by AFNOR. However, samples E2 and E5 had not shown any antimicrobial activity. Also, lot E6 and E9 presented average antimicrobial activities which are respectively 58.3 and $64.2 \%$. The study of the correlation between alcohol content and antimicrobial activity showed that the hydro-alcoholic products with an alcohol content between 60 and $80 \%$ also showed the best activities except for E2. This sample was in conformity with the alcohol content but not in conformity with the $\mathrm{pH}$ and the density, which seems to show the importance of the latter two parameters on the quality and efficacy of the hydro-alcoholic products. On the other hand, the E5 sample should no antimicrobial activity, which seems to support the hypothesis that there is an upper limit of alcohol content compatible with antimicrobial activity.

Indeed, alcohol acts by denaturing microbial proteins. This denaturation would be facilitated by the presence of water, and that is why alcohol solutions containing 60 to $80 \%$ alcohol have better antimicrobial activity than absolute alcohol and alcohols at very high concentrations that are less effective on germs (WHO, 2005; WHO, 2009).

\section{Conclusion}

This preliminary work revealed that hydro-alcoholic products with alcoholic degrees of between 60 and $80 \%$ also recorded the best antimicrobial activity, and the hydroalcoholic solution was more effective than hydro-alcoholic gels.

In the light of the results of this study, it would be appropriate to broaden and deepen the 
investigations by significantly increasing the number of samples and by carrying out as complete an analysis as possible. This should make it possible to obtain much more exhaustive data on the quality of hydroalcoholic products consumed in Senegal.

\section{COMPETING INTERESTS}

The authors declare that they have no competing interests.

\section{AUTHORS' CONTRIBUTIONS}

SA performed the various experiments and wrote the manuscript. $\mathrm{AD}$ and $\mathrm{MD}$ participated in the development of the paper. AM corrected the version of the paper. PMD; MMF; AD; $\mathrm{MF}$ and DF supervised the research. All authors read, improved the paper and accepted its final version.

\section{ACKNOWLEDMENTS}

We thank all the staff of the pharmacy of the hospital Aristide le Dantec and all the staff of the national laboratory of quality control of drugs.

\section{REFERENCES}

Chabot Daval MC. 2010. Evolution of the quality of hand friction with a hydroalcoholic solution: study among 270 hospital agents of the Nancy University Hospital. UHP - University Henri Poincaré.

Codell Carter K. Semmelweis I, Mayrhofer C. 1985. The Rise of Germ Theory. Médical History, 29: 33-53. DOI: $10.1017 / \mathrm{s} 0025727300043738$

Goetz M. 2004. Hydroalcoholic solutions: indications, advantages and disadvantages. Medicine Mal. Infect., 34: S124-S126. DOI: 10.1016/S0399-077X (04)90094-7.
Lejeune B, Aho Glele L, Barbut B, Ertzscheid Ma, Foegle J, Hajjar J, Lasheras A, Rogues Am, Seguier JC. 2008. L'hygiène des mains en question. Ektopic.

Meunier O, Kessler B, Garnier L, Burger S. 2015. The hydro-alcoholic solutions fluorescentes for quantifier the quality of hand friction. Alin\&as newsletter of the Clin and Arlin du Sud-Est.

Rouzic N, Tande D, Payan C, Garo B, Garre M, Lejeune B. 2011. Epidemiology of nosocomial MRSA infections at Brest University Hospital from January 1, 2004 to December 31, 2007. Impact of consumption of hydroalcoholic products and antibiotics. Pathol Biol Feb, 59(1): e1-5. DOI 10.1016/j.patbio.2010.10.001

Samake Sabiha Diallo. 2012. Thesis for the State Diploma of Doctor of Pharmacy. Implementation of the WHO multimodal strategy for the promotion of hand hygiene at chu Gabriel Tour in the Department of Medicine: state of the art. University of Sciences, Techniques and Technologies of BAMAKO,

Teyssou R, Koeck J-L Buisson Y.1997. Cutaneous flora. French review of laboratories.

Travkine M.2012. The interest of hydroalcoholic products in hospital, community, individual and family environments. Other, University of Lorraine.

WHO. 2009. Guidelines on Hand Hygiene in Health Care First Global Patient Safety Challenge Clean Care is Safer Care. WHO: Geneva.

WHO. 2005. Recommendations for Hand Hygiene in Health Care (Advanced Version). WHO: Geneva. 